\title{
Diaphragm pacing is associated with reduced survival in ALS patients with respiratory insufficiency
}

Respiratory failure is the principal cause of death in patients with amyotrophic lateral sclerosis (ALS), and noninvasive ventilation can prolong life by several months in these individuals. Preliminary studies have indicated that diaphragm pacing, combined with noninvasive ventilation, provides additional survival benefits; however, the results of a new

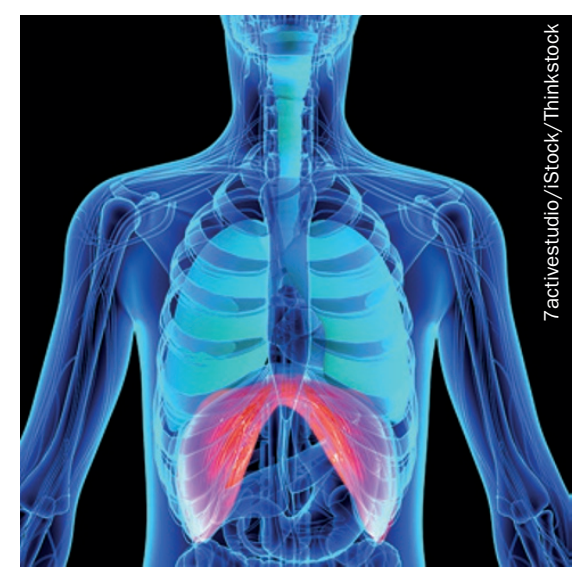

randomized controlled trial, known as the DiPALS study, suggest that this approach could actually be harmful.

The diaphragm pacing approach employed by the DiPALS study comprised a laparoscopically implanted device that rhythmically stimulated the phrenic nerve, thereby inducing contraction of the diaphragm. The DiPALS investigators randomly assigned 74 ALS patients with respiratory insufficiency to noninvasive ventilation alone or noninvasive ventilation plus diaphragm pacing.

The patients who received diaphragm pacing survived for a median of 11.0 months, compared with 22.5 months for those who received noninvasive ventilation alone. By the end of the 3-year study period, $76 \%$ of patients in the pacing group and $51 \%$ of patients in the noninvasive ventilation group had died.

These negative results did not seem to be attributable to immediate postoperative complications. However, previous research has indicated a link between surgical procedures and acceleration of the disease process in patients with ALS. The DiPALS authors suggest that the pacing itself could adversely affect vulnerable motor neurons, or cause excessive muscle fatigue.

The investigators conclude that diaphragm pacing cannot be recommended for routine use in patients with ALS at the onset of respiratory failure, and they warn against adopting a 'nothing to lose' approach in such patients. Nevertheless, the possibility remains that the intervention might be beneficial in a carefully selected subset of patients at an earlier stage of the disease process.

Heather Wood

Original article DiPALS Writing Committee, on behalf of the DiPALS Study Group Collaborators. Safety and efficacy of diaphragm pacing in patients with respiratory insufficiency due to amyotrophic lateral sclerosis (DiPALS): a multicentre, open-label, randomised controlled trial. Lancet Neurol. 14, 883-892 (2015) 